\section{Las relaciones coitales y la percepción de riesgo de adquirir ETS/SIDA en adultos jóvenes varones de Lima, Perú}

\author{
Sexual relations and the perception \\ of risk of acquiring STD/AIDS among \\ young adult men in Lima, Peru
}

Jesús L. Chirinos 1

Olga Bardales 2

María D. Segura 1
1 Instituto de Estudios de Población, Universidad Peruana Cayetano Heredia, Lima, Perú.

2 Programa Nacional contra la Violencia Familiar y Sexual, Ministerio de la Mujer y Desarrollo Social, Lima, Perú.

Correspondencia J. L. Chirinos Departamento Académico de Salud Pública, Facultad de Salud Pública y Administración Carlos Vidal Layseca, Instituto de Estudio de Población, Universidad Peruana Cayetano Heredia. Av. H. Delgado 430 , SMP, Lima-31, Perú jlchcu@gmail.com

\section{Abstract}

The purpose of this study was to identify men's co-responsibility in the negotiation process of dual protection, according gender, in their first and most recent sexual relationships, and their perception of the risk of STD/AIDS. We surveyed 750 males from 19 to 29 years of age in 2,250 randomly selected households in metropolitan Lima, Peru. The majority mentioned condoms as a contraceptive technique (95\%). Their first sexual relationships occurred with a female friend, with a stable partner, or with a known woman. Almost half used some type of protection, principally condoms in order to avoid pregnancy, but their consistent and correct use was low. For 39.5\%, the most recent sexual relationship was with a stable partner, a female friend or "known" woman, a wife, or a female co-resident. Dialogue about protection increased between the first and the most recent sexual relationships. The perception of risk of acquiring STD/AIDS was low, and they tended to use protection only with an "unknown partner".

Condoms; Sexual Behavior; Sexually Transmitted Diseases

\section{Introducción}

Los estudios sobre las masculinidades aparecen recientemente - desde los años 90 -, como parte de los estudios de género 1,2,3,4. El hombre ha estado presente tangencialmente en gran parte de los estudios realizados en mujeres y desde el punto de vista de la mujer. Últimamente, se genera una corriente orientada a estudiar a los varones desde su propia perspectiva y su involucramiento en la salud sexual y reproductiva propia, de la mujer y de la pareja, desde su situación y perspectiva de género $3,5,6,7$.

Algunos temas de importancia se han ido desarrollando desde la construcción social de la masculinidad y los factores que determinan la masculinidad hegemónica entre los varones que, desde nuestro punto de vista, es socialmente "fuerte" e individualmente "débil"; lo que afecta principalmente a las mujeres, al considerarlas más débiles $8,9,10$. Hay que tener en cuenta la influencia feminista en el desarrollo de los estudios de género y el entendimiento de la masculinidad hegemónica, los cambios actuales que se dan tanto en el modelo femenino y masculino social actual y su efecto en la relación de las parejas y de la emergencia de nuevos estilos de masculinidad y feminidad 8 .

Las formas de "ser hombres" y de "ser mujeres" son construcciones culturales históricas 11,12 y determinan los comportamientos de los seres humanos. En el caso de la identidad mas- 
culina, ésta se construye según Badinter 13, negando a la madre en la adolescencia, pues los jóvenes tienen que romper con el mundo privado (el hogar). Las formas de percibir los roles de género y el sistema genérico en una relación sexual influyen en esta negociación. Por ello se tiene que tomar en cuenta la construcción de las interacciones sociales de una pareja para comprender las distintas maneras de negociar que existen, dentro y fuera de las relaciones sexuales.

Por lo anterior, se diseñó una investigación poblacional en Lima y Callao en el Perú, como parte del estudio multicéntrico (que se basa en el modelo de creencias en salud 14 denominado "Multicenter Research Project in Peru, Cuba, Bolivia, and Argentina: Reality and Beliefs in the Sexual and Reproductive Decision-Making Process. Men's Perceptions and Behaviours"con el apoyo de la Organización Mundial de la Salud, durante los años 2000/2001. El objetivo general fue identificar corresponsabilidad del varón desde su propia sexualidad según género y señalar las motivaciones, cuidados y comportamientos dados durante la primera y la última relación sexual; así como, describir su percepción de riesgo de ETS/SIDA, y determinar las variables relacionadas en la asunción de medidas y conductas preventivas para su salud sexual y reproductiva.

\section{Población y métodos}

Se aplicó una encuesta (previamente mejorada por un estudio cualitativo y validada mediante un piloto) a 750 varones adultos jóvenes de 19 a 29 años, provenientes de 2.250 viviendas, seleccionados por muestreo aleatorio y polietápico. Los varones pertenecían a los estratos medio-alto, medio y medio-bajo de los Distritos de Lima Metropolitana, Perú en el 2001, de acuerdo al porcentaje de necesidades básicas insatisfechas, establecido por el Instituto $\mathrm{Na}$ cional de Estadística e Informática (INEI). Se midió también a través de la observación directa de los encuestadores en el hogar entrevistado, guiándose de una serie de criterios, relacionados con el material de la casa, puertas, techo, piso, y la posesión de diferentes muebles o bienes en el hogar. Se determinaron finalmente 3 niveles: "Alto/Medio Alto", "Medio", "Medio Bajo/Bajo".

En el cuestionario se inquirió sobre el conocimiento de anticoncepción y protección contra el SIDA, primera y última relación sexual, conocimiento de ETS y auto-percepción de riesgo. Los resultados fueron analizados a través de estadísticos descriptivos como fre- cuencias relativas, medias y desviaciones estándar, en las comparaciones se usó chi cuadrado y p de significancia, buscando asociaciones, de acuerdo con las limitaciones del estudio. Así como un análisis bivariado, para establecer las razones de momios (odds ratio) de prevalencia crudas (ORPc), con el objeto de realizar luego un análisis multivariado de regresión logística, utilizando como variable dependiente, por ejemplo, uso de condón (sí o no) y determinar el mejor modelo reducido que explica el ajuste de las variables (ORPA = OR de prevalencia ajustados) y llegar a modelos predictivos para la prevención.

\section{Resultados}

\section{Características sociodemográficas}

La media de edad de los entrevistados fue de 24 \pm 3 años. Dos tercios de ellos son solteros; casados el $12 \%$ y convivientes otro $12 \%$. El $21 \%$ de éstos tiene por lo menos un hijo, la mitad de ellos tuvo su primer hijo entre los 21-25 años y un tercio durante la adolescencia (Tabla 1).

\section{Sexualidad}

Los entrevistados señalaron que se puede evitar el embarazo utilizando el preservativo (95\%), píldoras (79\%), inyectables (57\%) y Dispositivo Intrauterino, 53\%. Para evitar el SIDA, el 74\% señala que usaría el condón, tendría una pareja estable/ sería fiel (65\%); muy pocos señalan la abstinencia espontáneamente, pero la reconocen cuando se le menciona. El 91,2\% de los entrevistados ha tenido relaciones sexuales.

- Primera relación sexual: en el 86,5\% de ellos, la edad media de la coitarquia fue a los 17 años \pm 2 años y se tuvo principalmente con la amiga; su pareja estable o una conocida. El 5\% indicó haber tenido relaciones coitales homosexuales o bisexuales. El 48\% hizo algo para cuidarse y la mayoría usó condón (480 jóvenes; $62 \%$ ). Al preguntar el motivo por el que no hablaron de cuidarse en la primera relación sexual, el 46,3\% respondió que no acostumbraba hablar del tema; el 31,3\%, que fue inesperado ("espontáneo" / "me sedujo"), el 7,7\% ignoraba acerca de los métodos anticonceptivos ("no sabían de ningún método" / "eran muy niños”), el 6,7\% no percibía el riesgo ("no pensaron en el peligro" / "habían bebido" / "era como un relajo" / "recién se conocían” / "era la primera vez" / "sabía que no iba a pasar nada” /"había confianza”), el $4,8 \%$, que ya se cuidaban ("era obvio que tenía que cuidarme" / "yo me cuido”), el 3,2\% porque 
Características de los encuestados.

\begin{tabular}{|c|c|}
\hline Característica & $\%$ \\
\hline Edad media de los encuestados & $24+3$ años \\
\hline \multicolumn{2}{|l|}{ Lugar de nacimiento } \\
\hline Lima - Callao & 83,0 \\
\hline Fuera de Lima & 17,0 \\
\hline \multicolumn{2}{|l|}{ Nivel socioeconómico } \\
\hline Alto/medio alto & 21,0 \\
\hline Medio & 43,0 \\
\hline Medio bajo/bajo & 36,0 \\
\hline \multicolumn{2}{|l|}{ Estado civil } \\
\hline Solteros & 74,5 \\
\hline Casados & 12,0 \\
\hline Conviviente & 11,0 \\
\hline Separados/divorciados/viudos & 1,6 \\
\hline \multicolumn{2}{|l|}{ Hijos } \\
\hline Con hijos & 21,0 \\
\hline Hijos & 79,0 \\
\hline \multicolumn{2}{|l|}{ Estudios } \\
\hline Primaria & 21,6 \\
\hline Secundaria & 47,2 \\
\hline Superior & 31,2 \\
\hline \multicolumn{2}{|l|}{ Condición de trabajo } \\
\hline Trabaja & 61,0 \\
\hline No trabaja & 39,0 \\
\hline \multicolumn{2}{|l|}{ Religión } \\
\hline Católico & 80,0 \\
\hline Otro & 20,0 \\
\hline
\end{tabular}

su pareja era prostituta, desconocida o que ya se cuidaba (Tabla 2 ).

- Última relación sexual: el 39,5\% tuvo su última relación coital con su pareja estable con quien no vive; $25 \%$ con una amiga o conocida, $27 \%$ con la esposa o conviviente. El 77,5\% menciona que en esa ocasión hicieron o usaron algo para cuidarse y las razones fueron: para cuidarse del embarazo $77 \%$, para cuidarse del SIDA 9,5\%, para evitar ambos el resto.

En esta relación sexual, el 57,7\% respondió que ya se cuidaba ("sabe que tiene que cuidarse para evitar ETS”/ “yo me cuido”), el 26,4\% respondió que no acostumbraban hablar del tema, el 11,5\%, que fue inesperado ("espontáneo" / "me sedujo"), el 4,3\% que no percibían el riesgo ("no pensaron en el peligro" / "habíamos
Tabla 2

Variaciones entre la primera y la última relación sexual.

Varones adultos jóvenes de Lima Metropolitana, Perú, 2001.

\begin{tabular}{|c|c|c|c|c|}
\hline \multirow[t]{2}{*}{ Característica } & \multicolumn{2}{|c|}{$\begin{array}{l}\text { Primera relación } \\
\text { sexual }(n=684)\end{array}$} & \multicolumn{2}{|c|}{$\begin{array}{l}\text { Última relación } \\
\text { sexual }(n=636)\end{array}$} \\
\hline & $\mathrm{n}$ & $\%$ & $n$ & $\%$ \\
\hline \multicolumn{5}{|c|}{ Edad del varón (años)* } \\
\hline$\leq 19$ & 615 & 90,0 & 30 & 4,7 \\
\hline$>19$ & 66 & 9,6 & 605 & 95,1 \\
\hline \multicolumn{5}{|c|}{ Edad de su pareja (años)* } \\
\hline$\leq 19$ & 388 & 56,7 & 112 & 17,6 \\
\hline$>19$ & 133 & 19,4 & 479 & 75,3 \\
\hline \multicolumn{5}{|c|}{$\begin{array}{l}\text { En esa ocasión hicieron algo } \\
\text { para cuidarse }{ }^{\star \star}\end{array}$} \\
\hline $\mathrm{Si}$ & 326 & 47,7 & 493 & 77,5 \\
\hline No & 345 & 50,4 & 140 & 22,0 \\
\hline \multicolumn{5}{|c|}{$\begin{array}{l}\text { En algún momento se habló } \\
\text { de cuidarse }\end{array}$} \\
\hline $\mathrm{Si}$ & 306 & 44,7 & 406 & 77,5 \\
\hline No & 360 & 52,6 & 227 & 22,0 \\
\hline
\end{tabular}

$\chi^{2} ; p=0,0000$

* Diferencia por no responde;

** Diferencia por no recuerda;

*** Diferencia por no sabe/no responde.

bebido" / "no nos importó" / "nos conocíamos"/ "era mi esposa" / "queríamos un hijo" / "ella estaba gestando”) (Tabla 2).

- Percepción de riesgo: el 58\% de los entrevistados cree que no corre riesgo de contagiarse de alguna de estas enfermedades y sus razones principales son: porque tiene pareja estable y son fieles $(50 \%)$, no tiene relaciones sexuales (26\%) y sólo el 5,5\% usa preservativo siempre. Los que dijeron que sí creen que corren riesgo señalaron como razones principales que se corre riesgo aunque se tenga una pareja estable o uno se cuide (32\%), hay muchas enfermedades $(31 \%)$ y porque falla el preservativo o la protección (10\%).

La percepción de correr riesgo de contagiarse de alguna enfermedad de transmisión sexual o VIH es menor entre los mayores de 24 años, porque tienen pareja estable o son fieles $(67 \%$ vs. 35\%) y entre los de 24 años o menos es porque no tiene relaciones sexuales ( $40 \% v s .10 \%)$. Sin embargo, son los mayores de 24 años los que en mayor proporción han sufrido de alguna ETS ( $81 \% v s .4 \%)$ acudiendo a un médico la mayoría de la veces ( $62 \% v s .53 \%)$, con una mayor proporción en los de 24 años o menos que acude al amigo (27\% vs. $8 \%$ ). 
- Precauciones en situaciones de riesgo: los entrevistados señalaron que en las siguientes situaciones tomarían precauciones para no contagiarse de SIDA: con una desconocida o una relación casual 99,1\%, una trabajadora sexual $99 \%$, una persona a la que no ama $98 \%$, una amante $96 \%$, un(a) conocido(a) $89 \%$, una persona virgen $58 \%$, una pareja estable con quien vive o novia $56 \%$ y una persona a la que ama mucho $52 \%$; no se cuidaría con su esposa $88 \%$.

\section{Resultados del análisis multivariado}

Al definir como variable dependiente el "uso del condón” se encontraron como variables que aumentaban su uso: no trabajar actualmente (ORP = 3,1; IC95\%: 2,1-4,5), si hizo o usó algo en la primera y última relación sexual (ORP = 2,289; IC95\%: 1,58-3,3; ORP = 2,68; IC95\%: 1,5$4,75)$ y si se habló de cuidarse en la última relación sexual (ORP = 2,75; IC95\%: 1,78-4,25).

Considerando como variable dependiente "hacer o usar algo en la última relación sexual", se encontró las siguientes variables relacionadas: pertenecer a la condición socioeconómica "media-media alta" (ORP = 1,6; IC95\%: 1,18$2,19)$; no creer que corren riesgo de adquirir alguna ETS (ORP = 2,38; IC95\%: 1,49-3,8), hablaron de cuidarse en la última $\mathrm{RS}(\mathrm{ORP}=8,47$; IC95\%: 5,25-13,65), hicieron o usaron algo en la primera RS (ORP = 2,3; IC95\%: 1,4-3,7); y usaron condón (ORP = 2,99; IC95\%: 1,67-5,38).

Cuando la variable dependiente fue "si creían que corren riesgo de tener una enfermedad de transmisión sexual", se encontraron variables relacionadas como: pertenecer a la condición socioeconómica "media baja-baja" $(\mathrm{ORP}=1,7$; IC95\%: 1,33-2,17), haber aprobado el último año (ORP = 1,49; IC95\%: 1,038-1,98); creer que hay situaciones en que se le debe pegar a los hijos (ORP = 2,66; IC95\%: 1,85-3,81) y no tener actualmente una novia o pareja estable (ORP = 1,92; IC95\%: 1,34-2,76).

\section{Discusión}

Para el caso de América Latina existe un modelo hegemónico de masculinidad, visto como un esquema culturalmente construido, en donde se presenta al varón como esencialmente dominante y que sirve para discriminar y subordinar a la mujer y a otros hombres que no se adaptan a este modelo 15 .

Esto implica que los varones se preocupen por el desempeño sexual con su pareja, por estar siempre listos a tener relaciones coitales, no preocupándose por las ETS, VIH/SIDA. Más les importa el tamaño del pene, ser el primero en la vida sexual de la mujer y tener relaciones sexuales muy frecuentes.

Vemos que la juventud, tiene una moratoria vital, que les hace percibir a la muerte como algo lejano, lo cual los lleva a comportamientos de riesgo. Además, la masculinidad implica negar lo femenino y lo homosexual a través de comportamientos heterosexuales dirigidos a la genitalidad, demostrándolo en todo tipo de relaciones sociales, en especial, en el grupo de pares.

En esta socialización podemos encontrar algunas claras ventajas para el varón algunas de las cuales, con el tiempo y su rigidez, se pueden ir transformando en un costo para su salud (y la de las mujeres y otros hombres). Ejemplo de esto son una mayor independencia, la agresividad, la competencia y la incorporación de conductas violentas y temerarias en aspectos tan diversos como la relación con vehículos, las adicciones, las relaciones familiares y la sexualidad 16 .

Ya se está encontrando una resignificación del papel del varón (como señalamos en el componente cualitativo de este estudio 17) al enfrentar cambios en los roles de género, como nuevas formas de ser mujer, como la que demuestra que tienen iniciativa sexual. Esta resignificación, creemos, pasa por un posible proceso de transición a las nuevas formas de masculinidades que pueden llevar a un varón a una hiper-virilidad sustentada en reforzar significados tradicionales sobre las características de lo masculino o por construir nuevos roles de masculinidad, criticando los roles tradicionales de la masculinidad hegemónica adulta.

Es significativo que en el uso del condón se mantengan prácticas entre los jóvenes como no usar el preservativo en todas las relaciones sexuales ni correctamente, teniendo aun poca percepción de riesgo. El uso del condón, como aquí se demuestra, tiene que ver con la forma de percibir a la "otra persona" y el tipo de relación que tengan. De esta manera, se usa el condón cuando no se conoce a la chica, mientras que no se usa con la enamorada, con una persona con quien se tiene confianza o tranquila y sólo se usa con el fin de evitar un embarazo y no una ETS. En cambio, cuando una mujer pide usar preservativo es vista como que ya ha tenido una experiencia sexual y se la estigmatiza, lo cual genera desconfianza y relaciones asimétricas en la negociación en el uso del condón.

Un dato importante es que la mayoría de los participantes cree que los jóvenes no usan el preservativo en su iniciación sexual, por dos 
motivos, porque éstas no son planeadas y desean sentir la sensación de una relación sexual sin condón, incrementando las prácticas sexuales de riesgo. Además de ello, percibir a una persona como saludable, cuando realmente no se está seguro de ello, condiciona una mayor percepción de riesgo.

La iniciación sexual de los varones entre 19 y 29 años se experimentó con diversas emociones, que respondían tanto a aspectos culturales, sociales, familiares e individuales. Muchas veces este evento suele ser esperado con ansiedad y/o temor generado por las expectativas sociales y personales referidas al género. Esto es importante, sobre todo si nos referimos al varón, puesto que se señala que en el varón existe una representación acerca de la iniciación sexual ligada a las percepciones y estereotipos acerca de ser hombre, y de su futuro desempeño sexual como tal 17.

La adolescencia es un período de cambios psicológicos, sociales y biológicos que crean condiciones para el ensayo de comportamientos y toma de decisiones relacionados en su mayoría con su sexualidad y que muchas veces pueden desencadenar en riesgos para su salud.

La iniciación sexual de los jóvenes en el presente estudio es con amigas o conocidas y no en los lenocinios. Una brecha importante a considerar es que, si además de esto, se tiene interiorizado que el condón se usa sólo con personas que no se conocen, vemos que su uso puede ser mínimo.

La negociación de los métodos anticonceptivos recién se da luego de un tiempo de empezada la relación coital. Además, consideran que es la mujer la que debe cuidarse, pues son las más afectadas. Es por ello que en una primera instancia no se constituye en un tema a ser considerado por el varón, colocándolos en una situación de riesgo.

Por otro lado, hemos encontrado que la iniciación sexual de casi la totalidad de los jóvenes varones de 19 a 29 años de Lima ocurrió tempranamente en la adolescencia, lo mismo que en su pareja; lo que puede significar un cambio en la práctica de la iniciación sexual de los varones comparada con otras generaciones, en donde el varón se iniciaba en su mayoría con personas mayores que ellos o trabajadoras sexuales.

Con respecto a la relación que tenía con su primera pareja sexual, en su mayoría los varones refieren en orden de prioridad que fue con una amiga, una pareja estable con quien no vivía, una conocida o una vecina. Esta práctica, nos puede llevar a reflexionar varias ideas; primero, representaría una respuesta de "protec- ción" de los adolescentes frente a las infecciones de transmisión sexual de esta época, incluyendo el SIDA; segundo, la presencia de una percepción en los adolescentes de "grupos de riesgo" (trabajadoras sexuales, homosexuales) llevándolos así, a evitar la iniciación sexual con ellas, por pensar que ellos son agentes de contagio, más no aquellas personas por las que sienten afecto y son conocidas. Esto los ha colocado de igual manera en una situación de riesgo, considerando que no existen grupos de riesgo, sino comportamientos de riesgo y su propia vulnerabilidad, al considerarse fuera del problema; y por último, se encuentra la opinión de que el sexo es "mejor" (seguro y placentero) si se realiza con una persona a la cual se está vinculado afectivamente (amiga, pareja, conocida, vecina) que guiado sólo por la "atracción física” o el placer o "apariencia” (trabajadora sexual).

En el tema de la anticoncepción en la gran mayoría no fue conversado dentro de la pareja, a pesar del alto conocimiento de qué hacer para prevenir un embarazo o una ETS/SIDA, lo cual los coloca en una situación de un probable embarazo no deseado y vulnerables al contagio de ETS. Esto indica que el tema de los anticonceptivos aún genera rechazo entre los adolescentes, generando un sentimiento de incomodidad al hablar del tema. Los que hicieron algo para cuidarse en su mayoría usaron el condón, siempre pensando más en el tema del embarazo que del contagio de las ETS; los que no hicieron nada indicaron que no tenían información, no tenían acceso a los métodos o por la confianza con su pareja o no romperla (supuestos o creencias).

En la última relación sexual, los jóvenes refieren en su gran mayoría que sí hicieron algo para cuidarse y fue para el embarazo y no para prevenir el SIDA, nuevamente.

Generalmente, las medidas de precaución para evitar el SIDA son tomadas por los jóvenes cuando establecen una relación con una persona a la cual no existe ninguna ligazón afectiva, sino de placer, atracción física o por comercio sexual, minimizando su precaución cuando están con una persona virgen, con una pareja "estable" o con su esposa, lo cual los coloca en una probabilidad de riesgo de igual manera, si es que no existe una relación fiel.

Por consiguiente, estos resultados nos llevan a un conjunto de sugerencias para ser tomadas en cuenta por los hacedores de políticas y que sean incorporadas en los diversos programas y estrategias para atender la salud sexual y reproductiva, especialmente en los varones. Se debe considerar que el componente de 
promoción y prevención es básico en cuanto a la información, comunicación y educación en sexualidad de los varones y su responsabilidad individual y recíproca que deben tener cuando asumen un comportamiento sexual que podría afectar profundamente en su vida.

La percepción de que una persona puede evitar el SIDA con el condón es un conocimiento bastante difundido, gracias a las campañas de comunicación tanto del estado como de las entidades no gubernamentales, y la mayor apertura por parte de la sociedad para tocar este tema; sin embargo, no se debe olvidar que también se menciona el tener una pareja estable y no estar con personas de riesgo, sobre lo cual debemos difundir mensajes de alerta para los jóvenes sobre el peligro que no visualizan. Como señalan mínimamente a la abstinencia y la fidelidad mutua, se tiene que trabajar más sobre los comportamientos de riesgo y la vulnerabilidad de los jóvenes, ampliando el espectro hacia la comunicación y la confianza mutua, llegando luego a la abstinencia y la fidelidad

\section{Resumen}

Para identificar la corresponsabilidad de los varones en el proceso de negociación de protección dual, según género en la primera y última relación sexual, y su percepción de riesgo de ETS/SIDA, se encuestó a 750 varones de 19-29 años en 2.250 viviendas de Lima Metropolitana, Perú, 2001, seleccionados aleatoriamente. La mayoría señaló principalmente el condón como una forma para evitar el embarazo (95\%). La primera relación coital fue con la amiga, su pareja estable o una conocida. Casi la mitad de ellos hizo algo para cuidarse, principalmente usó condón para evitar un embarazo, pero su uso consistente y correcto son bajos. El 39,5\% tuvo su última relación coital con su pareja estable con quien no vive, con amiga o "conocida", la esposa o conviviente. El diálogo sobre protegerse se incrementa entre la primera y la última relación coital. Su percepción del riesgo de adquirir ETS/SIDA es baja y se protegen sólo con parejas "desconocidas".

Condones; Conducta Sexual; Enfermedades Sexualmente Transmisibles mutua como prevención y manteniendo el uso efectivo del condón.

En el análisis multivariado de regresión logística se encontraron algunos modelos interesantes con lo que podríamos predecir los comportamientos de algunos varones.

Finalmente, y a manera de conclusión, diremos que los varones reconocen al condón como protección más para el embarazo que para las ETS; pero el uso consistente y correcto son bajos, por lo que deberíamos trabajar más en la doble protección del preservativo.

El diálogo con su pareja acerca de protegerse se incrementa entre la primera y la última relación sexual, lo que nos lleva a postular que se debe incidir más en las habilidades de comunicación y eliminar los mitos y creencias que existen entre varones y mujeres.

Existe un alto riesgo de adquirir ETS/SIDA al protegerse sólo con parejas "desconocidas", por lo cual hay que comprometernos a estudiar los comportamientos de riesgo de los más allegados y la vulnerabilidad de los jóvenes.

\section{Colaboradores}

J. L. Chirinos participó en el trabajo de campo, el procesamiento de la información y la redacción y corrección del presente manuscrito. O. Bardales participó en el trabajo de campo, así como en el procesamiento de la información y la redacción y corrección del presente manuscrito. M. D. Segura colaboró en el procesamiento de la información y en las correcciones del presente manuscrito.

\section{Agradecimientos}

Al apoyo financiero de la Organización Mundial de la Salud para el desarrollo del estudio. A los adultos jóvenes varones que participaron en el estudio. 


\section{Referencias}

1. Figueroa-Perea JG. Algunas reflexiones en la interpretación social de la participación del varón en los procesos de la salud reproductiva. Revista del Centro de Investigación y Estudios Avanzados de la Población de la Universidad Autónoma del Estado de México 2000; 25:59-82.

2. Figueroa-Perea JG, Liendro E. La presencia del varón en la salud reproductiva. In: Hardy E, Duarte MJ, Rodrigues E, editores. Ciencias sociales y medicina: perspectivas Latinoamericanas. Campinas: Universidade Estadual de Campinas; 1995. p. 193-226.

3. Mundigo A. Re-conceptualizando el rol de los hombres en la era post-Cairo. In: Seminario sobre Hombres, Formación Familiar y Reproducción. Buenos Aires: Unión Internacional para el Estudio Científico de la Población/Centro de Estudios de Población; 1998. p. 7-27.

4. Stycos JM. Men, couples, and family planning: a retrospective look. New York: Population and Development Program, Cornell University; 1996.

5. Keijzer B. La masculinidad como factor de riesgo. In: Seminario Fecundidad y el Ciclo Vital del Varón en la Era de la Declinación de la Fecundidad. Zacatecas: Unión Internacional para el Estudio Científico de la Población; 1995.

6. Collumbien M, Hawkes S. Missing men's messages: does the reproductive health approach respond to men's sexual health needs? Cult Health Sex 2000; 2:135-50.

7. Hawkes S, Hart G. Men's sexual health matters: promoting reproductive health in an international context. Trop Med Int Health 2000; 5:37-44.

8. Connell R. Masculinities. London: Polity Press; 1995.

9. Kaufman M. Los hombres, el feminismo y las experiencias contradictorias del poder entre los hombres. In: Valdés T, Olavarría J, editores. Masculinidad/es. Santiago de Chile: Facultad Latinoamericana de Ciencias Sociales; 1997.
10. Kimmel M. La masculinidad como Homofobia: miedo, vergüenza y dolor. In: Valdés T, Olavarría J, editores. Masculinidad/es. Santiago de Chile: Facultad Latinoamericana de Ciencias Sociales; 1997.

11. Fuller N. Reflexiones sobre el machismo en el Perú. In: Valdés T, Olavaria J, editores. Masculinidades y equidad de género en América Latina. Santiago de Chile: Facultad Latinoamericana de Ciencias Sociales; 1998. p. 258-68.

12. Badinter E. XY La identidad masculina. Santa Fé de Bogotá: Editorial Norma; 1993.

13. Becker M, Maiman L. Models of health-related behavior. In: Mechanic D, editor. Handbook of health, health care and the health professions. New York: The Free Press; 1983. p. 5-12.

14. Hernández I. Identidades étnicas subordinadas e identidades masculinas hegemónicas. In: Valdés T, Olavaria J, editores. Masculinidades y equidad de género en América Latina. Santiago de Chile: Facultad Latinoamericana de Ciencias Sociales; 1998. p. 218-29.

15. Keijzer B. La masculinidad como factor de riesgo. In: Seminario Taller sobre Masculinidades. Lima: Population Council; 1999.

16. Cáceres C. Jóvenes varones en Lima: dilemas y estrategias en salud mental. In: Valdés T, Olavaria J, editores. Masculinidades y equidad de género en América Latina. Santiago de Chile: Facultad Latinoamericana de Ciencias Sociales; 1998. p. 158-74.

17. Chirinos JL, Bardales O, Cabezudo C. Realidades y creencias en los procesos de toma de decisiones sexuales y reproductivas. Percepciones y comportamientos de los hombres. Lima: Organización Mundial de la Salud; 2001.

Recibido el 30/Mar/2005

Versión final presentada 30/Jun/2005

Aprobado el 08/Jul/2005 